\title{
Efficacy of fexofenadine in isolated rat tracheas*
}

\author{
Wen-Sen Lai',2, Yuan-Yung Lin', Yueng-Hsiang Chu', Chih-Hung Wang', \\ Hsing-Won Wang ${ }^{1,3}$ \\ Department of Otolaryngology-Head and Neck Surgery, Tri-Service General Hospital, National Defense Medical Center, \\ Taipei, Taiwan \\ ${ }^{2}$ Department of Otolaryngology-Head and Neck Surgery, Taichung Armed Forces General Hospital, Taichung, Taiwan \\ ${ }^{3}$ Graduate Institute of Clinical Medicine and Department of Otolaryngology, College of Medicine, Taipei Medical University, \\ Shuang Ho Hospital, New Taipei City, Taiwan
}

\author{
Rhinology 51: 376-380, 2013 \\ DOl:10.4193/Rhino12.213
}

*Received for publication:

December 10, 2012

Accepted: April 25, 2013

\section{Summary}

Objectives: Histamine is an important chemical mediator in both nasal and bronchial inflammation in patients with allergic rhinitis and asthma. The effect of histamine receptor-1 antagonists on nasal mucosa in vivo is well known, however, the effect on tracheal smooth muscle has rarely been explored. The purpose of this study was to determine the effects of fexofenadine on isolated tracheal smooth muscle in vitro.

Methods: Six tracheal strips were used for each experiment, and one untreated strip served as a control. We examined the effectiveness of fexofenadine on isolated rat tracheal smooth muscle by testing the effect on: 1) tracheal smooth muscle resting tension; 2) contraction caused by $10^{-6} \mathrm{M}$ methacholine as a parasympathetic mimetic; and 3) electrically induced tracheal smooth muscle contractions.

Results: The results indicated that addition of methacholine caused the trachea to contract in a dose-dependent manner. The addition of fexofenadine at a dose of $10^{-4} \mathrm{M}$ elicited a significant relaxation response compared to $10^{-6} \mathrm{M}$ methacholine-induced contraction. There were no detectable changes in the peak tension of electrical field stimulation-induced contractions in the fexofenadine group.

Conclusion: High concentrations of fexofenadine had an anti-cholinergic effect. In addition to diminishing histamine-mediated allergic symptoms, fexofenadine may have a potentially therapeutic implication in alleviating asthma-related symptoms due to reducing methacholine-induced contractions of tracheal smooth muscle though these aspects were not studied.

Key words: fexofenadine, histamine receptor-1 antagonist, tracheal smooth muscle, in vitro study, anti-cholinergic effect

\section{Introduction}

Histamine is an important chemical mediator in both nasal and bronchial inflammation in patients with allergic rhinitis (AR) and asthma. In the past decade, extensive research has supported the concept of a united airway in which AR and asthma can be considered the same disease affecting different parts of the airway. Histamine, cysteine-containing leukotrienes, helper T (Th2) cytokines (IL-4, IL-5, IL-13, granulocyte-macrophage colony-sti- mulating factor), proteases, and chemokines released from mast cells and basophils appear to be central to the pathogenesis of allergen-induced airway inflammation ${ }^{(1)}$. Histamine release after immunological challenge plays a key role in this inflammatory response, and results in smooth muscle contraction, vasodilation, mucus hypersecretion, and oedema. The pathophysiology of these two disease states suggests that $\mathrm{H} 1$-antihistamines have a potentially therapeutic implication in AR and asthma. 
The second-generation antihistamines, including azelastine, cetirizine, desloratadine, fexofenadine, levocetirizine and loratadine have been developed to reduce or eliminate the sedation and anticholinergic adverse effects found with the older histamine receptor antagonists, as well as for a wider application in allergic and upper respiratory diseases. Fexofenadine hydrochloride is a nonsedating $\mathrm{H} 1$ antihistamine that has been approved for the therapy of allergic diseases. Since asthma is an allergic disease, it is reasonable to explore the role of fexofenadine in asthma. The tracheal smooth muscle plays an important role in reducing pulmonary function as it becomes contracted, causing extensive airway narrowing during an acute attack of asthma ${ }^{(2,3)}$. Hence, the effect of fexofenadine on tracheal smooth muscle deserves further exploration.

In light of our understanding of the effects of fexofenadine in tracheal constriction or relaxation, a simple in vitro model was applied to rat tracheas. The technique used was developed from a previously described method ${ }^{(4)}$, in which 5-mm strips of rat tracheas were suspended in a tissue bath containing $30 \mathrm{ml}$ Krebs solution ${ }^{(5,6)}$. One end of the strip was attached to a steel plate and the other to an isometric transducer and a steel plate. A passive tension of $0.3 \mathrm{~g}$ was applied to the strips. The purpose of this study was to determine the effects of fexofenadine on isolated tracheal smooth muscle in vitro.

\section{Materials and methods}

Sample

This study was approved by the Animal Experiment Review Board of Taipei Medical University (LAC-99-0299). The chemicals used were of the highest purity available. Pure fexofenadine hydrochloride powder was obtained through the courtesy of Sanofi-Aventis US LLC (Bridgewater, NJ, US). The other chemical reagents were obtained from Sigma (St Louis, MO, USA). We first tested methacholine with regards to tracheal contraction. Eighteen rats were anesthetized by intraperitoneal administration of pentobarbital $(45 \mathrm{mg} / \mathrm{kg}$ ) and two pieces of trachea about $5 \mathrm{~mm}$ in length were removed from each rat. The tracheal specimens were mounted using two steel plates and submersed in a $30 \mathrm{ml}$ muscle bath at $37^{\circ} \mathrm{C}$. The bath was filled with $30 \mathrm{ml}$ Krebs solution consisting of (mmol/l) $\mathrm{NaCl}$, (118); $\mathrm{KCl},(4.7)$; $\mathrm{CaCl}_{2^{\prime}}$ (2.5); $\mathrm{MgSO}_{4} \cdot 7 \mathrm{H}_{2} \mathrm{O},(1.2) ; \mathrm{KH}_{2} \mathrm{PO}_{4^{\prime}}$ (1.2); $\mathrm{NaHCO}_{3^{\prime}}$ (25.0); and glucose, (10.0). The upper side of the tracheal ring was attached to a Grass FT-03 force displacement transducer (AstroMed, West Warwick, RI, USA) by using a steel plate and a 3-O silk ligature. The other side of the ring was fixed to a steel plate attached to the bath. A passive tension of $0.3 \mathrm{~g}$ was applied to the strips and subsequent changes in tension were recorded continuously using Chart V4.2 software (PowerLab, ADInstruments, Colorado Springs, CO, USA). Preliminary tests showed that a tracheal strip immersed in the bath solution and then used for subsequent experiments did not contract when basal tension was applied. Before drug assays were conducted, isolated tracheas were equilibrated in the bath solution for 15 to 30 minutes with continuous aeration with a mixture of $95 \% \mathrm{O}_{2}$ and $5 \% \mathrm{CO}_{2}$. Stepwise increases in the amount of drugs used were employed to study the contraction or relaxation responses of the tracheal strips. All drugs were administered by adding a defined volume of stock solution to the tissue bath solution. In each experiment, one untreated strip served as a control.

\section{Electrical field stimulation}

Electrical field stimulation (EFS) $(5 \mathrm{~Hz}, 5$-ms pulse duration, at a voltage of $50 \mathrm{~V}$, trains of stimulation for $5 \mathrm{~s}$ ) was applied to the trachea ring with two wire electrodes placed parallel to the trachea strip and connected to a direct-current stimulator (Grass S44, Quincy, MA, USA). An interval of 2 minutes was imposed between each stimulation period to allow for recovery from the response. Stimulation was applied contiguously to the trachea at $37^{\circ} \mathrm{C}$.

Assessment of fexofenadine administration

Six tracheal strips were used for each experiment, and one untreated strip served as a control. The effect of fexofenadine administration was assessed by the effect on: 1) tracheal smooth muscle resting tension; 2 ) contraction caused by $10^{-6} \mathrm{M}$ methacholine (a parasympathetic mimetic) by examining postsynaptic events such as muscle-receptor blockade, enhancement, and second messengers; and 3) electrically induced contractions, i.e. whether electrical stimulation of the tissue caused parasympathetic nerve remnants in the trachea to release the transmitter acetylcholine. If there is interference with transmitter release, electrical stimulation does not cause contraction, and therefore presynaptic events were seen more easily with this procedure.

\section{Statistical analysis}

The mean tension between two different concentration agents was recorded in the basal tension and methacholine experiments, and the mean of the EFS peak between two different concentration agents in the EFS experiments. Data are presented as mean values and standard deviations (SD). Differences between mean values were compared using the Student t-test. Differences were assumed to be significant at $\mathrm{p}<0.05$.

\section{Results}

The degree of contraction or relaxation of the tracheal rings was estimated from the tension applied to the transducer. Tracheal contraction induced by a small dose of methacholine was easily detected, and the tissue remained in a contracted state until the drug was rinsed from the tissue.

Addition of the $\mathrm{H} 1$ antagonist, fexofenadine, to the tracheal 
rings elicited a negligible effect on basal tension. It resulted in relaxation of the trachea when introduced after the addition of a constricting agent such as $10^{-6} \mathrm{M}$ methacholine (Figure 1). Low doses of fexofenadine resulted in a mild effect on contraction, while higher doses significantly relaxed the trachea smooth muscle (Figure 1, 2). At $10^{-8} \mathrm{M}$ fexofenadine, the tension was $98.7 \pm 0.91 \%$ of the control values, and at $10^{-4} \mathrm{M}$ fexofenadine, the tension was $69.32 \pm 2.64 \%$ (Figure 2). The difference in tension between $10^{-8} \mathrm{M}$ and $10^{-4} \mathrm{M}$ fexofenadine was statistically significant $(p<0.05)$. However, fexofenadine could not inhibit EFS-induced spike contractions (Figure 3, 4). The peak tension of the tracheal rings evoked by EFS upon the addition of $10^{-8} \mathrm{M}$ fexofenadine was $98.51 \pm 1.82 \%$, whereas at $10^{-4} \mathrm{M}$ fexofenadine the peak was $84.35 \pm 14.50 \%$ (Figure 4). There was no significant difference between these responses.

\section{Discussion}

Fexofenadine, the active metabolite of terfenadine, is a selective histamine $\mathrm{H} 1$ receptor antagonist that does not cross the blood brain barrier and appears to display some anti-inflammatory properties. $\mathrm{H} 1$ antihistamines have been shown to attenuate the symptoms associated with the early- and late-phase clinical development of allergic diseases, as evidenced by a $50 \%$ inhibition of bronchoconstriction within one hour after allergen challenge (7). Other studies have also concluded that $\mathrm{H} 1$ antihistamines have the greatest effect in patients who have chronic allergic inflammation and hyper-responsiveness of the upper and lower airways, with a reduction in the clinical use of $\beta 2$-adrenergic

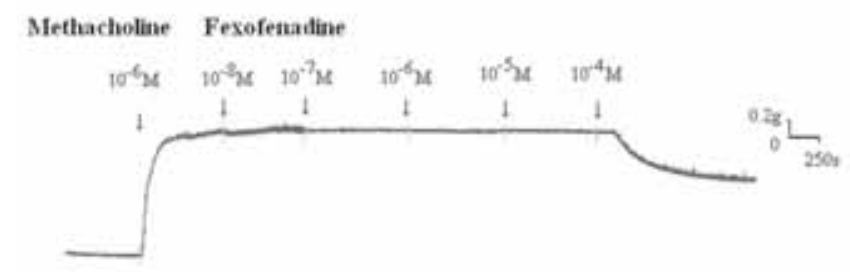

Figure 1. Original recording of the effects of fexofenadine on $10^{-6} \mathrm{M}$ methacholine-induced contraction of a rat trachea.

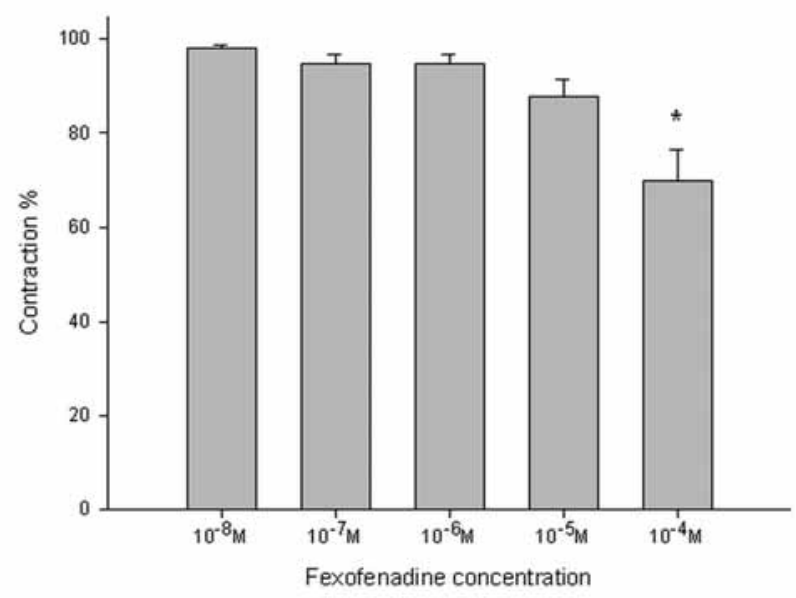

Figure 2. Effects of fexofenadine on 10-6 M methacholine-induced contraction (contraction area calculated at $100 \%$ with no addition of fexofenadine) of a rat trachea. The difference in tension between 10-8 M and $10-4 \mathrm{M}$ fexofenadine was statistically significant $(\mathrm{p}<0.05)$. Results are shown as mean $\pm \operatorname{SD}(n=6)$.

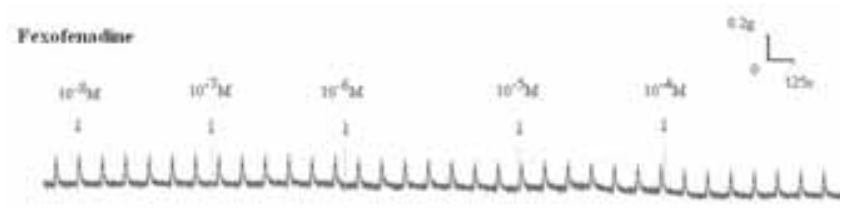

Figure 3. Original recording of the effects of fexofenadine on electrically induced tracheal smooth muscle contractions. Higher doses of fexofenadine did not decrease the spike contraction induced by electrical field stimulation.

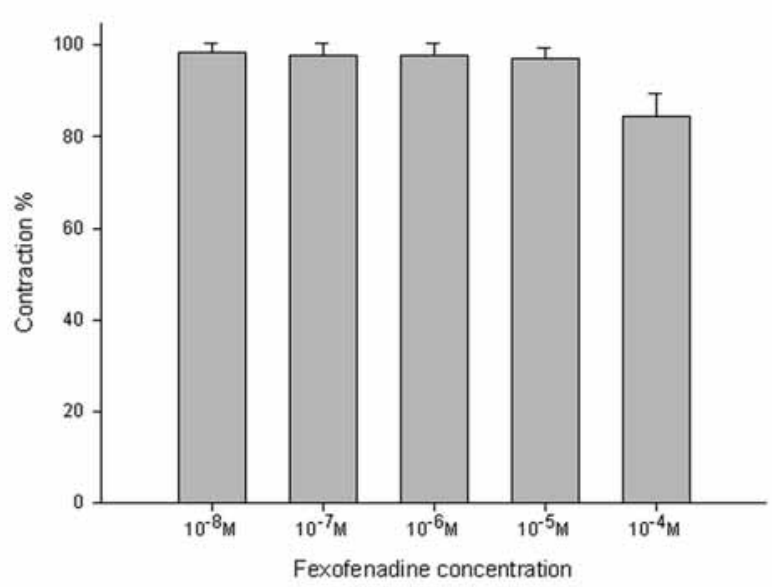

Figure 4. Effects of fexofenadine on electrically-induced tracheal smooth muscle contractions (contraction area was calculated at $100 \%$ with no addition of fexofenadine). There was no detectable difference in the responses of these tracheal rings compared with the addition of 10-8 $\mathrm{M}$ fexofenadine. Results are shown as mean $\pm S D(n=6)$. 
agonists, to relieve asthmatic and coryzal symptoms and improve pulmonary function ${ }^{(8,9)}$. Poorly controlled allergic rhinitis may contribute to worsening asthma control, and the treatment of postnasal drip has been shown to improve coughing in patients with asthma. Fexofenadine has been shown to significantly reduce the inflammatory markers when given as add-on therapy to corticosteroid-treated atopic asthmatic patients ${ }^{(10)}$. Clinically, there is a relationship between AR and asthma such that treatment of the upper airway has been shown to benefit lower airway disease.

There is evidence to suggest that $\mathrm{H} 1$-receptor antagonists may have to be administered at several times the doses used in the treatment of AR to alleviate asthma-related symptoms (11). In addition, it has been reported that the newer second-generation antihistamines may be useful in the treatment of asthma (6). In the current in vitro study, we attempted to evaluate the anticholinergic effect of fexofenadine in isolated rat tracheas. An intact tracheal ring was an important component of our technique, as an intact tracheal ring is much more representative of a physiological setting than smooth muscle strips ${ }^{(4,5,11)}$. In contrast to previous studies ${ }^{(3)}$, our test was simpler and more robust than those in which tracheal rings were destroyed. Furthermore, the cholinergic contracting agent tested in this study is commonly used for research purposes. It is particularly noteworthy that fexofenadine-induced relaxation of the tissue was dependent on prior partial contraction of the smooth muscle using methacholine. Thus, it seems to be possible to assay the effects of common drugs and agents supposedly responsible for relieving asthma. Fexofenadine hydrochloride is approved in the US for twice-daily dosing (60 mg) or once-daily dose $(180 \mathrm{mg}$ ) in the treatment of AR. When the concentration of fexofenadine reached $10^{-4} \mathrm{M}$ in the tracheal smooth muscle, muscle contraction was reduced. We previously conducted similar experiments for cetirizine ${ }^{(6)}$ and azelastine ${ }^{(12)}$, which had the same effects on isolated tracheal smooth muscle at $10^{-5} \mathrm{M}$ and $10^{-4} \mathrm{M}$, respectively. This indicates that high concentrations of $\mathrm{H} 1$-antihistamines have an anti-cholinergic effect in relaxing tracheal smooth muscle.

In the past decade, extensive research has supported the concept of a united airway in which AR and asthma can be considered the same disease affecting different parts of the airways. Histamine release after immunological challenge plays a key role in this inflammatory response that results in smooth muscle contraction, vasodilation, mucus hypersecretion, and oedema. The anti-inflammatory and bronchodilator effects of $\mathrm{H} 1$-antihistamines may be concentration dependent. In a double-blind, placebo-controlled study on patients with AR and mild-to-moderate perennial asthma, treatment with cetirizine
$20 \mathrm{mg}$ over 26 weeks was found to significantly reduce the severity of wheezing, shortness of breath, chest tightness, and nocturnal asthma, as well as rhinitis symptoms ${ }^{(8)}$. A recent review also advocated that second-generation $\mathrm{H} 1$-antihistamines with the highest therapeutic ratio and the least likelihood of adverse events at high doses should be used in patients with $A R$ and comorbid asthma ${ }^{(13)}$. Our results also provide evidence that fexofenadine can reduce the methacholine-induced contraction of tracheal smooth muscle, and the anti-cholinergic effect may contribute to a reduction in asthma-related symptoms.

EFS is a common experimental tool that activates the nerve terminals within the tissue being tested and induces the release of endogenous neurotransmitters, thereby triggering the contraction of smooth muscle. EFS-induced spike contraction of isolated canine nasal mucosa has been shown to be mediated by sympathetic innervation ${ }^{(14)}$. In the current study, EFS-induced spike contraction of the tracheal smooth muscle was believed to be the result of stimulation of parasympathetic innervation, the same mechanism as in an asthma attack. It had no significant effect on the parasympathetic component of EFS-induced contraction in the trachea, even though the concentration of fexofenadine was increased. This finding suggests that fexofenadine does not antagonize the parasympathetic innervation responsible for trachea smooth muscle contraction.

There are some limitations to this study. First, the tracheal strips used were crude preparations that contained cartilage and tracheal smooth muscle. The smooth muscle of the trachea appeared to be the main tissue component responsible for contraction, as the other components (epithelium, glands, connective tissue, nerves, and cartilage) did not contract to a significant extent. The contractile response observed in this study was probably an aggregate of the responses of various types of muscle tissue. Second, the isolated tracheal preparations used in our experiments were excised from rats, as it is difficult to obtain human tissue for such studies. Third, this was an vitro study, and the responses may be more complicated in in vivo conditions.

\section{Conclusion}

Second-generation antihistamines are highly selective for the $\mathrm{H} 1$ receptor and bind to it with high affinity. According to our results, the degree of drug-induced tracheal contraction or relaxation was dose dependent, and a high concentration of fexofenadine was found to have an anti-cholinergic effect. As well as diminishing the histamine-mediated allergic symptoms, fexofenadine may have a potentially therapeutic implication in alleviating asthma-related symptoms due to reducing methacholine-induced contraction of tracheal smooth muscle though these aspects were not studied. 


\section{Acknowledgement}

None.

\section{Authorship contribution}

Study conception and design: LWS, CYH, WCH, WHW Acquisition of data: LWS, CYH, WHW

\author{
Analysis and interpretation of data: LWS, LWW, CYH \\ Drafting of the manuscript: LWS, LWW, CYH, WCH, WHW \\ Critical revision: WCH, WHW
}

\section{Conflict of interest}

The authors declare no conflicts of interest.

\section{References}

1. Baena-Cagnani CE, Desloratadine activity in concurrent seasonal allergic rhinitis and asthma. Allergy. 2001; 56 Suppl 65: 21-27.

2. Beny JL, Pacicca C, Bidirectional electrical communication between smooth muscle and endothelial cells in the pig coronary artery. Am J Physiol. 1994; 266 (4 Pt 2): H1465-1472.

3. Kellner J, Tantzscher J, Oelmez H, et al., Mechanisms altering airway smooth muscle cell Ca+ homeostasis in two asthma models. Respiration. 2008; 76: 205-215.

4. Gonzalez O, Santacana GE, Effect of low temperature on tracheal smooth muscle contractile and relaxing responses evoked by electrical field stimulation. P R Health Sci J. 2001; 20: 237-244.

5. Wang HW, Wu CC, Effects of oxymetazoline on isolated rat's tracheal smooth muscle. Eur Arch Otorhinolaryngol. 2008; 265: 695698.

6. Kao CH, Chu YH, Wang HW, Effects of cetirizine on isolated rat's tracheal smooth muscle. Eur Arch Otorhinolaryngol. 2009; 266: 753-757.

7. Twentyman OP, Ollier S, Holgate ST, The effect of $\mathrm{H} 1$-receptor blockade on the development of early- and late-phase bronchoconstriction and increased bronchia responsiveness in allergen-induced asthma. J Allergy Clin Immunol. 1993; 91: 1169-1178.

8. Aaronson DW, Evaluation of cetirizine in patients with allergic rhinitis and perennial asthma. Ann Allergy Asthma Immunol. 1996; 76: 440-446

9. Corren J, Harris AG, Aaronson D, et al., Efficacy and safety of loratadine plus pseudoephedrine in patients with seasonal allergic rhinitis and mild asthma. J Allergy Clin Immunol. 1997; 100 (6 Pt 1): 781-788.

10. Lee DK, Jackson CM, Haggart K, Lipworth BJ, Repeated dosing effects of mediator antagonists in inhaled corticosteroid-treated atopic asthmatic patients. Chest. 2004; 125: 1372-1377

11. Gelfand EW, Role of histamine in the pathophysiology of asthma: immunomodulatory and anti-inflammatory activities of H1-receptor antagonists. Am J Med. 2002; 113 Suppl 9A: 2S-7S

12. Wang HW, Chou YL, Chu YH, Azelastine nasal spray inhibiting parasympathetic function of tracheal smooth muscle. Rhinology. 2010; 48: 211-215.

13. Bachert C, Maspero J, Efficacy of second- generation antihistamines in patients with allergic rhinitis and comorbid asthma. J Asthma. 2011; 48: 965-973.

14. Wang HW, Jackson RT, Do cholinergic neurons directly innervate nasal blood vessels? Rhinology. 1988; 26: 139-146.

Hsing-Won Wang, MD, PhD

Graduate Institute of Clinical Medicine and Department of Otolaryngology

College of Medicine

Taipei Medical University Shuang Ho Hospital, Jhonghe, New Taipei City Taiwan

Tel: +886-2-87927192

Fax: +886-2-87927193

E-mail:w0512n@ms15.hinet.net 